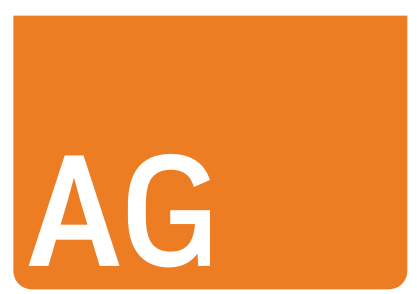

\title{
Die Aktiengesellschaft
}

Zeitschrift für deutsches, europäisches und internationales Aktien-, Unternehmensund Kapitalmarktrecht

\section{Herausgeber:}

Prof. Dr. Heinz-Dieter Assmann, LL.M., Universität Tübingen, E-Mail: assmann@jura.uni-tuebingen.de · Prof. Dr. Mathias Habersack, Universität München, E-Mail: mathias.habersack@jura.uni-muenchen.de

in Verbindung mit VorsRiBGH Prof. Dr. Ingo Drescher, Karlsruhe · Prof. Dr. Volker Emmerich, Bayreuth . VizepräsBVerfG Prof. Dr. Stephan Harbarth, LL.M., Karlsruhe · Prof. Dr. Jens Koch, Bonn · Prof. Dr. Hans-Joachim Mertens, Königstein · Prof. Dr. Dr. h.c. Uwe H. Schneider, Mainz/Frankfurt a.M. · RA Prof. Dr. Jochen Vetter, München · Prof. Dr. Dr. h.c. Wolfgang Zöllner, Tübingen

\section{Ständige Mitarbeiter AG-Report:}

RA Prof. Dr. Michael Arnold, Stuttgart · Prof. Dr. Walter Bayer, Jena · Dipl.-Verw. Wiss. Marianne Gajo, Spaichingen · Dr. Thomas Ledermann, Hamburg · Dr. Franz-Josef Leven, Frankfurt a.M. · Dr. Stefan Mai, Frankfurt a.M. · RA Dr. Stefan Mutter, Düsseldorf · Markus Rieger, Wolfratshausen · WP Prof. Dr. Eberhard Scheffler, Hamburg · Dipl.-Vw. Christoph Schlienkamp, Düsseldorf · Andreas Schmidt, München · Prof. Dr. Dr. h.c. Uwe H. Schneider, Mainz/Frankfurt a.M. · RAin Daniela Weber-Rey, LL.M., Frankfurt a.M. · RA Dr. Jochen Weck, München

\section{Steuer-Journal:}

Streck Mack Schwedhelm, Rechtsanwälte/Fachanwälte für Steuerrecht, Köln/Berlin/München

\section{Inhalt}

\section{Aufsätze}

\section{Dr. Philipp Maximilian Holle - Aufbewahrung von Unterlagen durch das Aufsichtsratsplenum und Aufsichtsratsmitglieder}

Die Tätigkeit von Aufsichtsräten ist in den vergangenen Jahren immer weiter ausgebaut und professionalisiert worden. Dementsprechend verarbeiten, verwalten und produzieren Aufsichtsräte und ihre Mitglieder eine wachsende Vielzahl von Informationen. Zugleich haben ein immer realer werdendes Haftungsrisiko und die Einführung der Business Judgment Rule dazu beigetragen, dass Aufsichtsräte ein immer größeres Augenmerk darauf legen, ihre Tätigkeit minutiös zu dokumentieren, um sich gegebenenfalls gegen den Vorwurf verteidigen zu können, eine uninformierte Entscheidung getroffen zu haben. Was bislang freilich nur vereinzelt beleuchtet worden ist, ist die sich an ein solches Gebaren früher oder später zwangsläufig anschließende Frage, ob, auf welche Art und Weise und über welchen Zeitraum hin der Aufsichtsrat beziehungsweise einzelne Aufsichtsratsmitglieder verwendete und hervorgebrachte Unterlagen aufzubewahren haben. Dem soll in diesem Beitrag nachgegangen werden.

\section{Steuer-Journal}


Inhalt

\section{Kommentar}

RA Dr. Ralph Schilha - ludex calculat: Richtiges Zählen von Leiharbeitnehmern im Rahmen der Anwendungsschwellenwerte für die Unternehmensmitbestimmung - Kommentar zu BGH v. 25.6.2019 - II ZB 21/18, AG 2019, 798

Wann sind Leiharbeitnehmer bei der Ermittlung der für die Anwendbarkeit der Unternehmensmitbestimmung relevanten Schwellenwerte zu berücksichtigen? Diese Zählfrage war bislang höchst umstritten und ist vor dem Hintergrund der praktischen Bedeutung der Arbeitnehmerüberlassung von zunehmend tatsächlicher Relevanz. Der BGH hatte nun die seltene Gelegenheit, eine für die Unternehmensmitbestimmung relevante Streitfrage höchstrichterlich zu entscheiden. Der Beitrag würdigt diese Entscheidung in ihren Folgen für die Praxis und lenkt den Blick auf die noch offenen und damit virulenten Fragen für ihre Anwendung im Einzelfall.

Univ.-Prof. Dr. Sebastian Mock, LL.M.(NYU)/Wiss. Mitarb. Benjamin Goltner, LL.B. (WU) - Stellung und Haftung des fehlerhaft bestellten besonderen Vertreters - die Grundsätze des fehlerhaften Bestellungsverhältnisses im Stresstest - Kommentar zu LG Heidelberg v. 24.7.2019 - 120 8/19 KfH, AG 2019, 804

Der besondere Vertreter stellt, selbst nach über zehnjähriger Entwicklung, Literatur und Rechtsprechung noch immer vor enorme Herausforderungen. Dabei geht es in solchen Fallkonstellationen zumeist um zwei Fragenkomplexe: einerseits stellt sich die Frage der Wirksamkeit der Bestellung des besonderen Vertreters mit all ihren Konsequenzen und andererseits um den Umfang seiner Kompetenzen. Das diesem Beitrag zugrundeliegende Urteil des LG Heidelberg zeigt eindrucksvoll die Schwierigkeiten, die in beiden Problembereichen auftreten können. Die folgende Besprechung soll daher beide Themenkomplexe beleuchten und damit insbesondere die Grundsätze des fehlerhaften Bestellungsverhältnisses in Bezug auf besondere Vertreter fortentwickeln.

\section{Rechtsprechung}

Insolvenzrecht: Cash Pooling in der Insolvenz des Konzerns, Abtretung eines

Rückgewähranspruchs

Genossenschaftsrecht: Gesetzliche Zuständigkeit zur Kündigung des Vorstands einer Genossenschaft aus wichtigem Grund

(BGH, Urt. v. 2.7.2019 - II ZR 155/18).....

Mitbestimmung: Berücksichtigung von Leiharbeitnehmern im Rahmen des § 1 MitbestG

(BGH, Beschl. v. 25.6.2019 - II ZB 21/18)

Aktienrecht: Rechtsstellung und Haftung eines mangelhaft bestellten besonderen Vertreters der Gesellschaft

(LG Heidelberg, Urt. v. 28.8.2019 - 120 8/19 KfH - Fall der Gelita AG [nrkr.]).

\section{AG Report}

Rechts-Report | Finanzmarktaufsicht

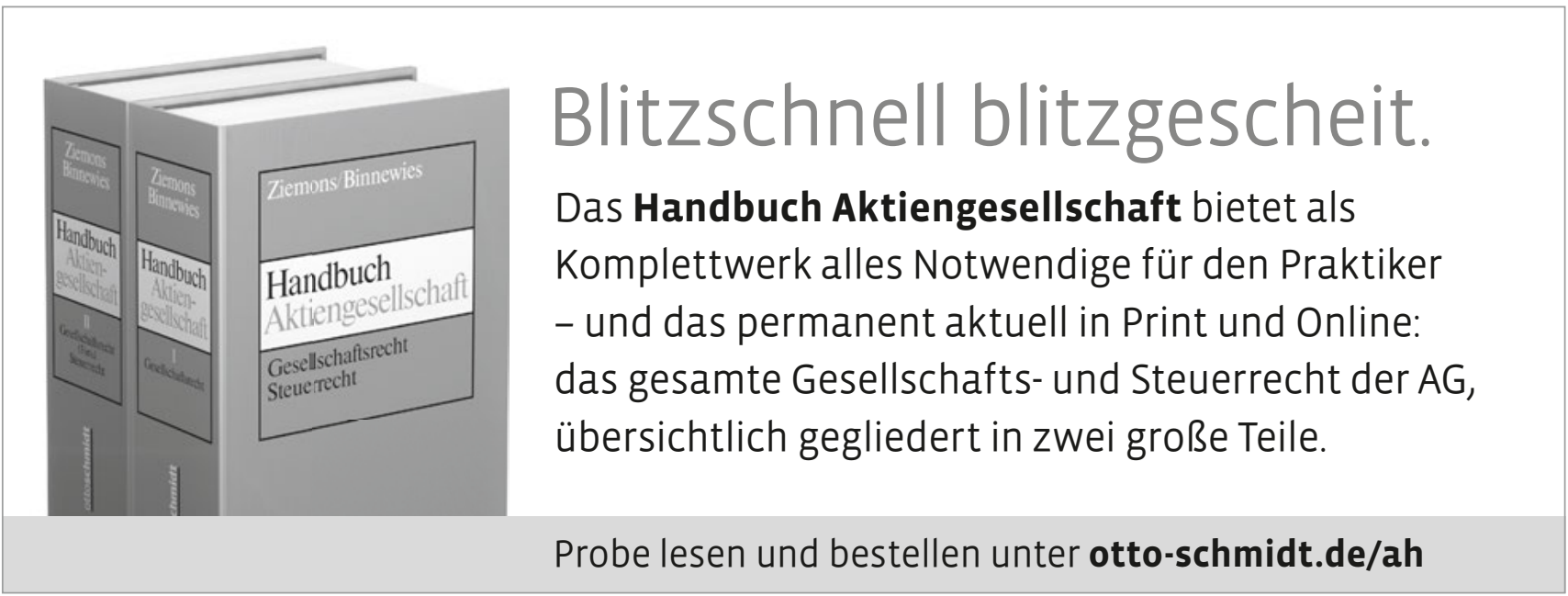


Inhalt

Rechts-Report | Anlegerschutz

Verjährung der Nachforderung des Kunden bei falscher Zinsberechnung in Prämiensparverträgen (Marlen Träber).

Kapitalmarkt-Report | Zahlen, Fakten, Entwicklungen

Legal Tech: Große Umfrage zur Digitalisierung der Anwaltschaft gestartet (Anwalt-Suchservice)..... R310

\section{Kapitalmarkt-Report | Börse}

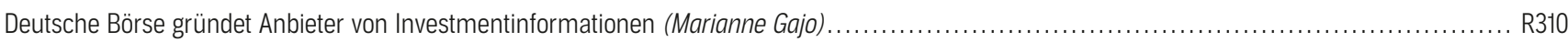

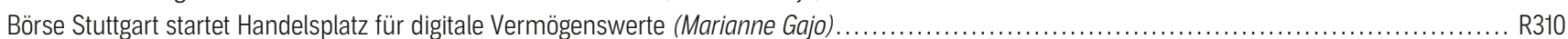

Börse Luxemburg und Euwax starten LuxXPrime für Anleihen (Marianne Gajo) .................................................. R311

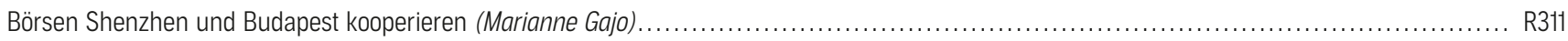

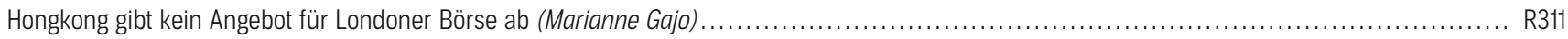

Dubai und Climate Bonds Initiative unterstützen grünen Sukuk-Markt (Marianne Gajo) ............................................ R312

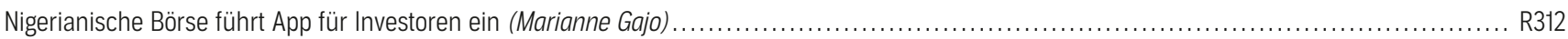

Branchen- und Unternehmens-Report | Branchen-Nachrichten

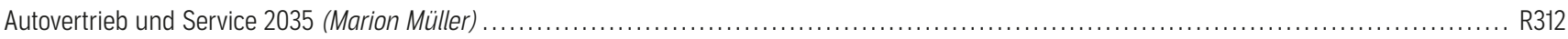

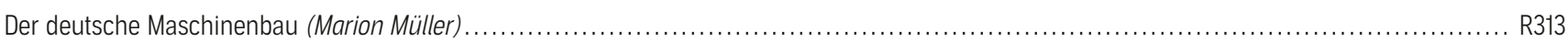

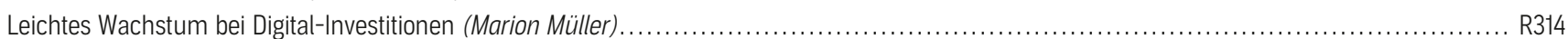

Branchen- und Unternehmens-Report | Jahresabschlüsse

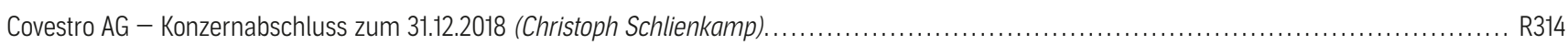

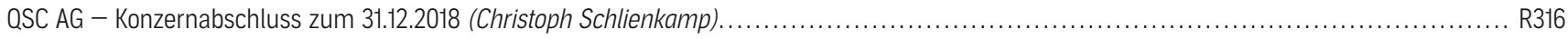

\section{Bibliothek}

Zeitschriftenspiegel (Katharina Melkko)......

\section{Wussten Sie schon ...}

Im Beratermodul AG haben Sie Zugriff auf das Online-Archiv Ihrer Zeitschrift. In der Zeitschriften-App lesen Sie aktuelle Beiträge auf Ihrem

Smartphone. Bei Fragen zu Ihren Freischaltcodes wenden Sie sich gerne an den Kundenservice: Telefon 0221 / 93738 -997 oder

E-Mail an kundenservice@otto-schmidt.de.

\section{Beratermodul}

> Kartellrecht
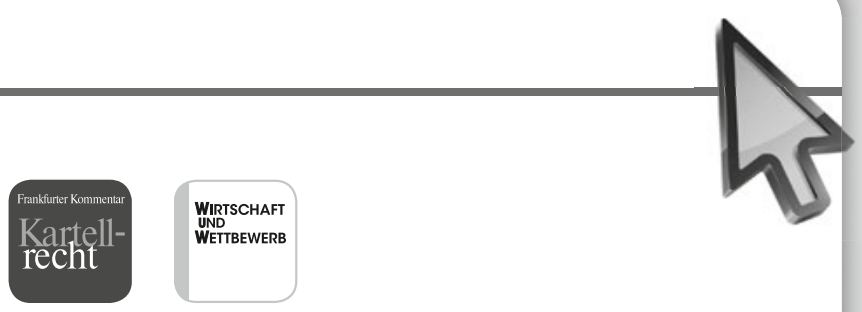